\title{
Broadband Vibration Energy Harvesting from Underground Trains for Self-Powered Condition Monitoring
}

\author{
Hailing $\mathrm{Fu}^{1}$, Wenzhe Song ${ }^{2}$, Yong Qin ${ }^{3}$, Eric M. Yeatman ${ }^{4}$ \\ ${ }^{I}$ Wolfson School of Mechanical, Electrical and Manufacturing Engineering, Loughborough University, \\ Leicestershire, United Kingdom \\ ${ }^{2}$ Department of Aeronautics, Imperial College London, London, United Kingdom \\ ${ }^{3}$ State Key Laboratory of Rail Traffic Control \& Safety, Beijing Jiaotong University, Beijing, China \\ ${ }^{4}$ Department of Electrical and Electronic Engineering, Imperial College London, London, United Kingdom
}

\begin{abstract}
A broadband vibration energy harvester tailored for self-powered condition monitoring of underground trains is proposed and developed using mechanical non-linearity and integrated multi-mode vibration. A datadriven approach is adopted for harvester design using operational vibration data on a train bogie. The harvester is designed to be unobtrusive while exhibiting good performance in harvesting energy over a wide bandwidth. In this work, the on-site vibration data are first analysed with the design goals identified. Then, a broadband harvester is proposed, implemented and evaluated. The harvester consists of a pre-stretched hosting beam and a group of micro-beams with repulsive magnetic forces on their free ends. A multiple vibration-mode harvester with non-linear dynamics is obtained in such a design. This harvester exhibits good performance over a broad bandwidth in frequency sweep and pseudo-random tests, illustrating its capability in self-powered condition monitoring applications.
\end{abstract}

\section{Introduction}

Rail transport is an essential transportation tool, but experiences regular failures of various types, resulting in passenger inconvenience, huge maintenance costs and occasionally catastrophic damage, injuries or deaths [1]. Increasing the railway system smartness is a solution, in which railway systems can identify their conditions autonomously and be maintained based on these conditions [2]. However, many challenges need to be addressed first, of which power supply is an important one. As distributed electrical power is unavailable on most rail tracks and freight train cars, and battery replacement is impractical, an alternative is needed. Energy harvesting, a technology converting environmental sources such as light, heat, or vibrations into electricity, provides a solution [3]. Extensive efforts have been made in effective energy harvesting [4-6], but harvester design driven by real operational vibration data from railway systems is little explored.

Another challenge for railway vehicle vibrations is the broadband nature of this energy source. As the train cars are complex systems with many sources of vibration, the vibration frequency components are abundant. Therefore, energy harvesters targeted at this energy source should be able to perform well across the vibration bandwidth. Different methods are available for broadband energy harvesting, such as bi-stability [7], frequency up-conversion [8] and multi-mode vibrations [9]. In this work, we combined the non-linear dynamics with multi-mode vibration in a unique energy harvester design based on on-site operational data.

\section{Broadband Vibration Harvester Design}

In order to understand the characteristic of vibration conditions in typical underground trains, on-site data were collected from different locations on a train bogie under operational conditions. Fig. 1 illustrates the operational data acquired on an underground train bogie travelling between two stations in Shanghai over 2 minutes. Fig. 1(a) shows the path of the traveling train. The velocity of the train is below $80 \mathrm{~km} / \mathrm{s}$ with significant changes, as shown in Fig. 1(b). The correlated acceleration on different parts of the train bogie are plotted in Fig. 1(c)-(e) with the peak acceleration below $\pm 4 \mathrm{~g}$.

To understand the frequency distribution of the vibrations, a frequency-domain analysis is conducted with the results illustrated in Fig. 2. Fig. 2(a) gives the results of a continuous wavelet transform, showing the variation of the dominant vibration frequencies in time and frequency domains. Fig. 2(b) presents the frequency domain results of the vibration signal from Fig. 1(d). The vibration frequency components are widely distributed from 0 to $150 \mathrm{~Hz}$. To harness this type of energy, a harvester covering this frequency range is necessary. Based on the above analysis, in order to effectively harness vibration energy sources from such a train bogie, it is important for the harvester to perform well over a broad bandwidth. 

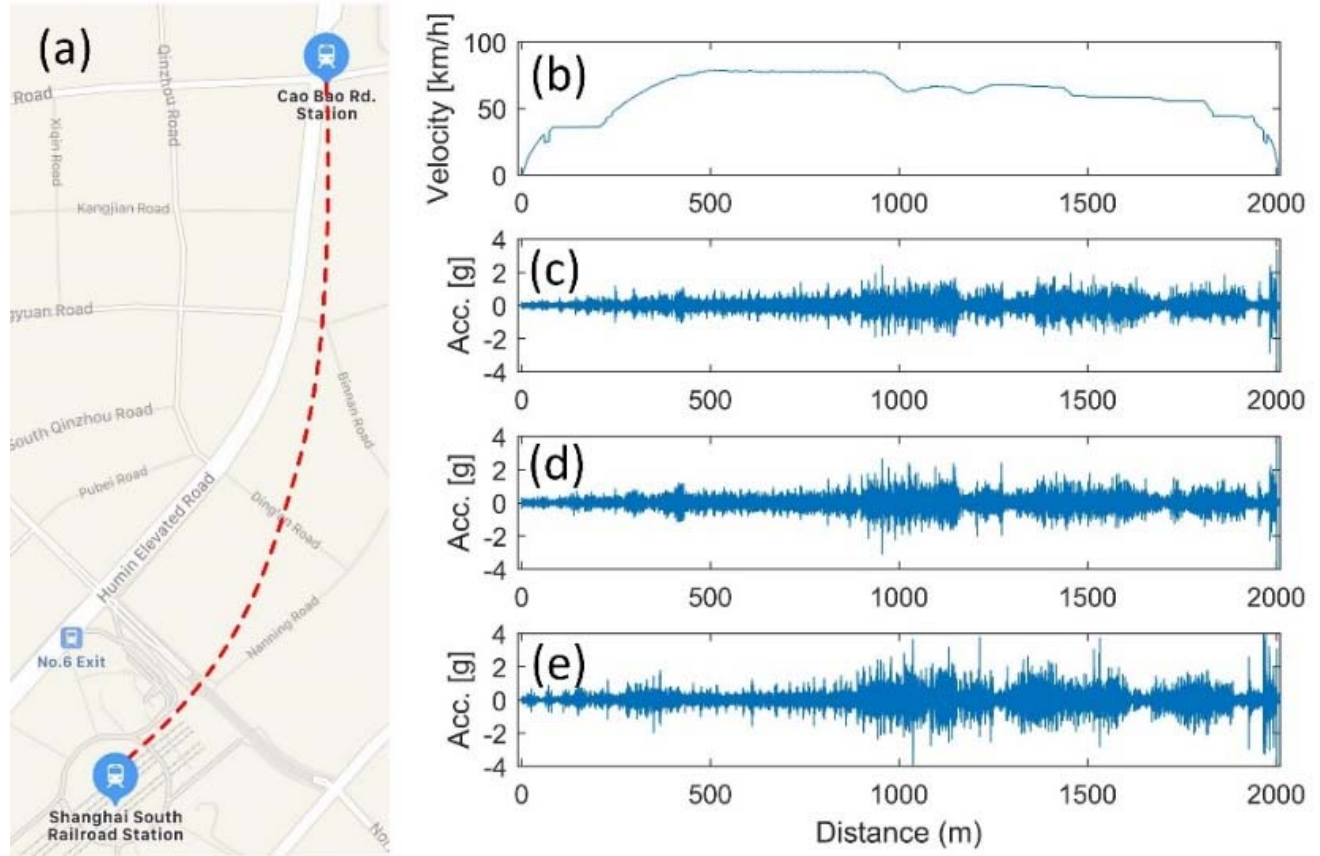

Fig. 1: On-site operational vibration data collected from different locations on a train bogie. (a) Path of the underground train in Shanghai during which the data were collected; (b) train velocity versus mileage (about $2 \mathrm{~km}) ;(\mathrm{c}),(\mathrm{d})$ and (e) train bogie acceleration on different locations.
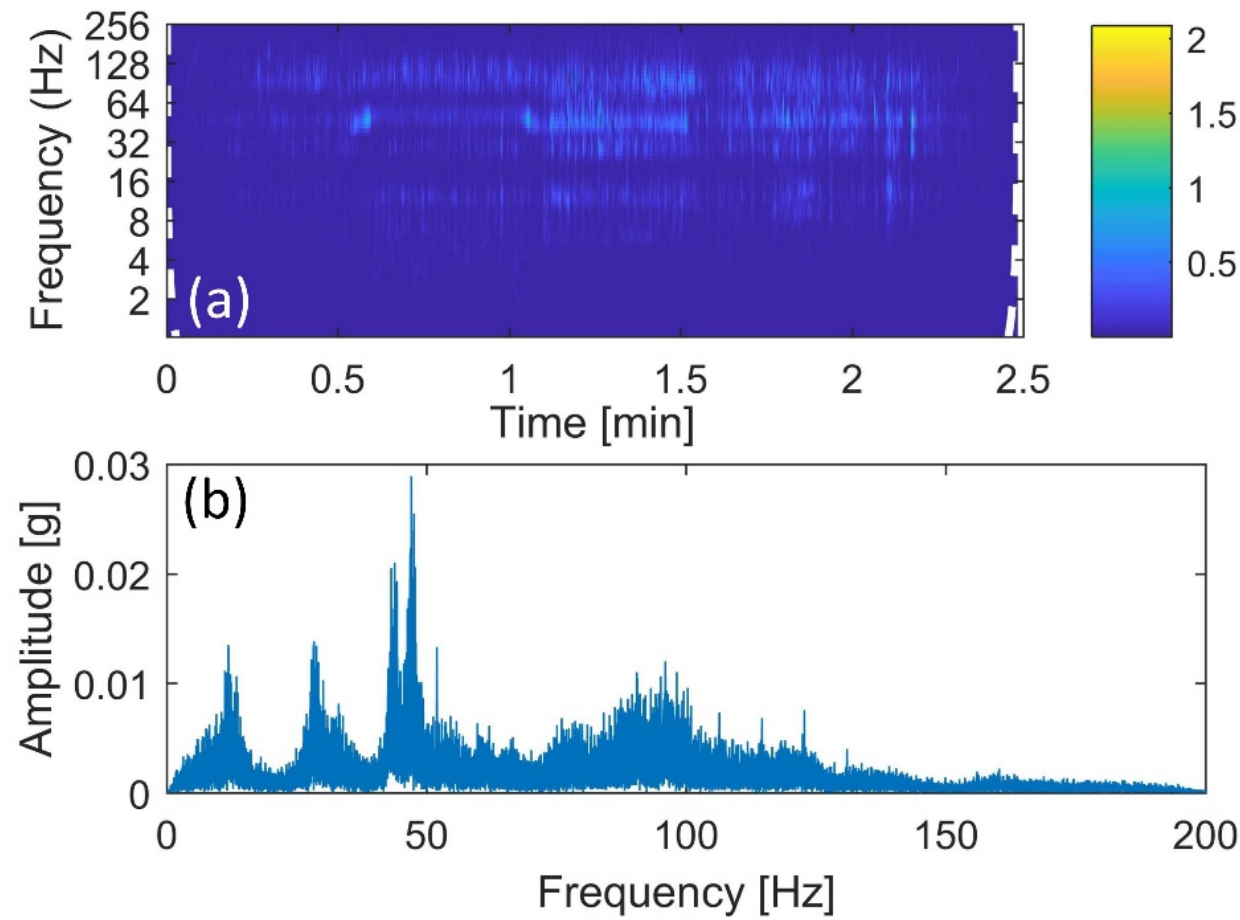

Fig 2: Frequency domain characteristics of on-site operational data (Fig. 1(d)). The vibration energy is mainly distributed between 10 to $130 \mathrm{~Hz}$ broadly. Multiple main frequency components co-exist during the whole path $(2 \mathrm{~km})$, as shown in Fig. 2(a).

A broadband harvester is, therefore, proposed using mechanical non-linearity and integrated multi-mode vibration, as shown in Fig. 3. A hosting beam in tension with multiple internally deflected micro-beams is designed, as shown in Fig. 3(c). The hosting beam is in tension, acting as a hardening oscillator. The microbeams are deflected due to the repulsive forces between the magnets mounted on their tips. A bistable configuration is obtained with the middle beam either resting above the hosting beam or below. The beams in the middle of the group of three micro-beams are piezoelectric beams. Electricity can be generated when these 
beams undergo changes in strain under vibration. Multi-mode vibration can be obtained due to the combination of different structures. Fig. 3(a) and (b) provide an indication of how this design can be mounted on a train bogie. A group of 24 harvesters are mounted on the train bogie as an example. The number of harvesters will be determined by the space available and the energy required for sensing in practice. The mounting location will also be partially determined by the locations of sensing modules.

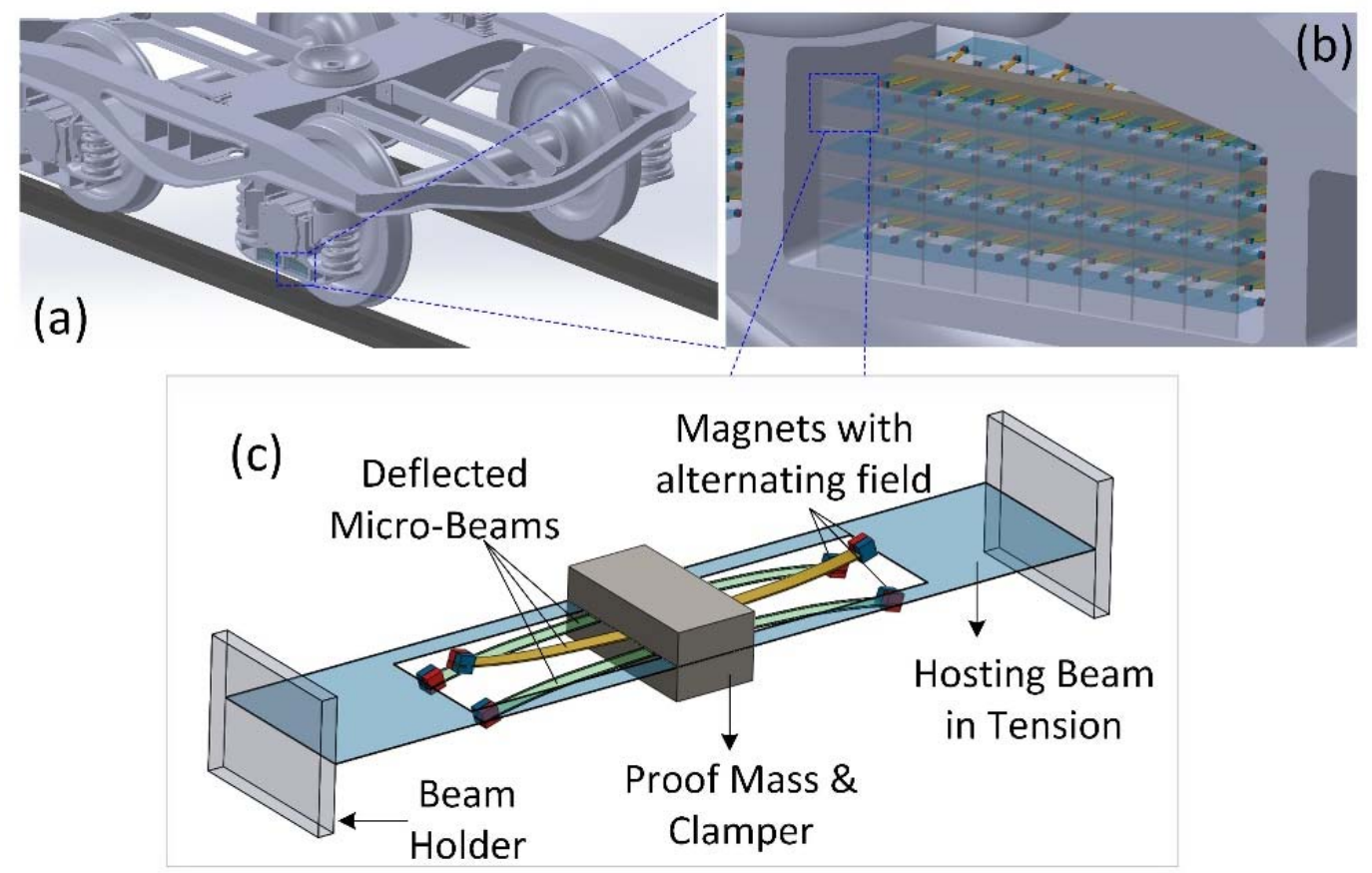

Fig 3: Broadband energy harvester design and its application in self-powered condition monitoring. (a) Harvester installation in train bogie suspension system; (b) details showing a group of 24 harvesters installed; (c) broadband energy harvester design using non-linearity and multi-mode vibration.

\section{Experimental Set-up and Results}

The prototype and the experimental setup are illustrated in Fig. 4. The hosting beam was implemented on a plastic thin plate. A 3D-printed proof mass was placed in the middle of the hosting beam. The proof mass also acts as the holder for the micro-beams. Magnets were mounted on the free ends of the micro-beams with the magnetic forces being repulsive. Piezoelectric beams were placed as two of the micro-beams in the middle. The whole structure in Fig. 4(a) is mounted on a fixture and eventually on a shaker, as shown in Fig. 4(b).

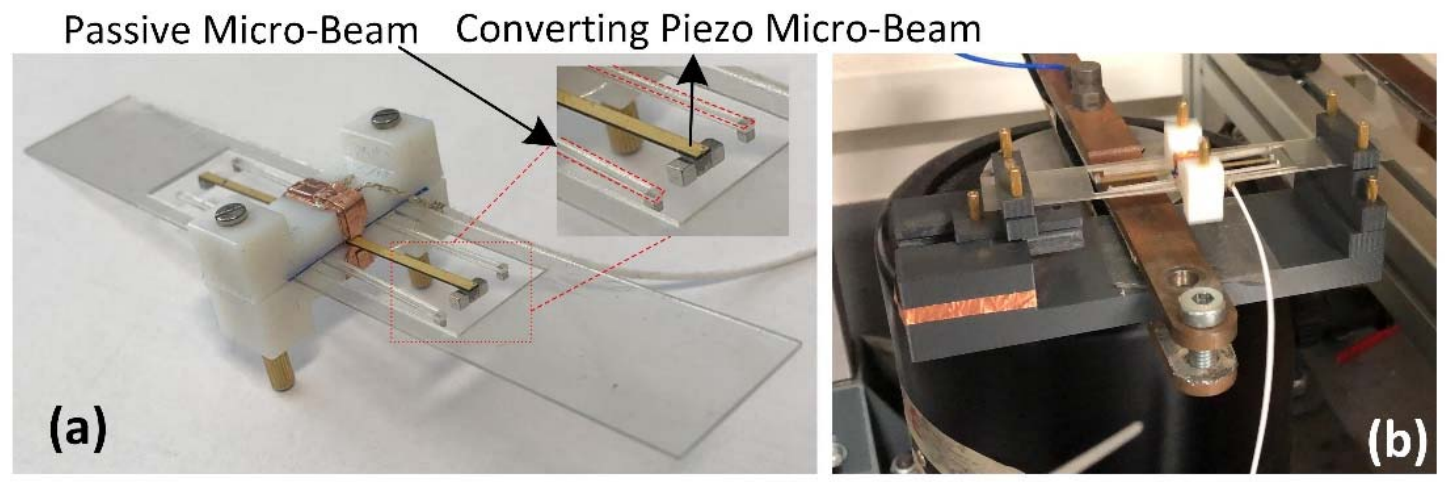

Fig. 4: Harvester prototype and experimental set-up. (a) harvester with two piezoelectric beams $(26 \times 1.5 \times$ $0.3 \mathrm{~mm}$, yellow colour) in the middle and four passive beams on the side; (b) harvester clamped on a holder and tested on a shaker. (Proof mass: $1.1 \mathrm{~g}$ )

Frequency sweep tests were conducted for different excitation levels. Figure 5 shows the frequency up- and down-sweep tests between 0 to $150 \mathrm{~Hz}$ under acceleration of $1.3 \mathrm{~g}$. The non-linear and multi-mode vibration 
dynamics can be observed. Several vibration peaks appear over this range at around $50 \mathrm{~Hz}, 100 \mathrm{~Hz}$ and 130 Hz. The voltage output was measured on a $1 \mathrm{M} \Omega$ resistor.

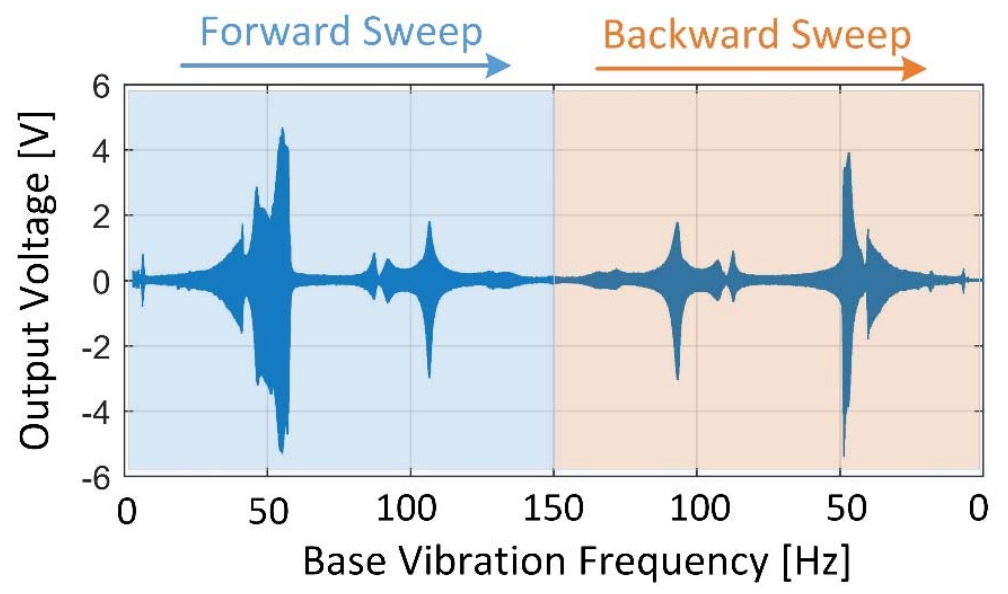

Fig 5: Frequency sweep test, showing the broad bandwidth of the harvester in the range the railway train operates. The harvester (1 PZT beam) was tested under $1.3 \mathrm{~g}$ with a $1 \mathrm{M} \Omega$ load resistor between $5 \mathrm{and} 150 \mathrm{~Hz}$.

To understand the dynamics of the system under different operation conditions, the harvester was tested under different acceleration levels in the frequency sweep test. The RMS voltages are presented in Fig. 6 . The broadband nature of this harvester is illustrated for different excitation conditions. It can be observed that with the increase of excitation levels, more vibration modes can be excited with increased output power. For example, a peak appears at a low frequency around $20 \mathrm{~Hz}$ under an acceleration of $2.5 \mathrm{~g}$. These results show that the harvester is capable of covering the vibration energy within the range of the main frequency components of the train bogie vibration. The output power can be obtained using $P=V^{2} / R$. A maximum RMS output power, $16 \mathrm{uW}$, can be obtained at round $50 \mathrm{~Hz}$.

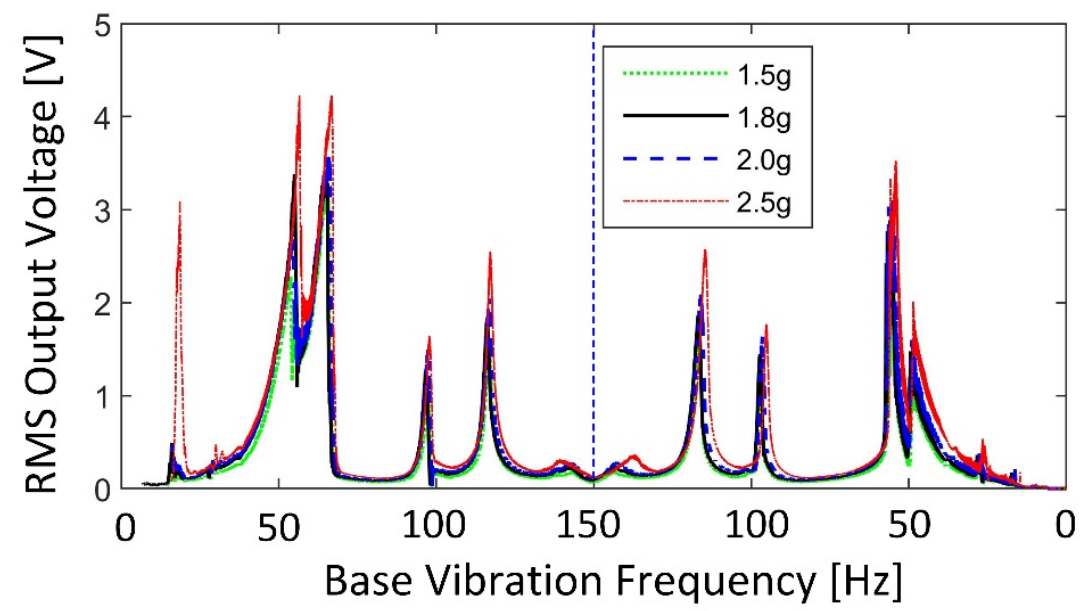

Fig 6: RMS output voltage versus base vibration frequency for different base acceleration levels. The bandwidth and output amplitude increase with the increase of the excitation level (1 M $\Omega$ load).

In practice, the vibration of a train can be random with multiple frequency. In order to examine the robustness of the harvester under random conditions, the harvester was then tested on a pseudo-random condition from 5 to $400 \mathrm{~Hz}$ at $0.0058 \mathrm{~g}^{2} / \mathrm{Hz}$. Fig. 7(a) gives the output voltages of the harvester under pseudorandom vibration. Fig. 7(b) shows the frequency distribution of the output voltage. The broadband and multimode vibration features are illustrated. The results validate the broadband characteristic again, confirming the feasibility of this design in effective harnessing broadband vibrations in underground trains.

Another critical concern about energy harvesting is the amplitude of output voltage, as a rectification circuit is necessary for converting AC output into DC for sensing applications. As shown in Fig. 7(a), the output voltage $(-4 \mathrm{~V}$ to $3 \mathrm{~V})$ is more than the typical forward voltage for Schottky diodes $(150 \mathrm{mV}-450 \mathrm{mV})$. This voltage loss has to be considered if no other better rectification method can be provided. 

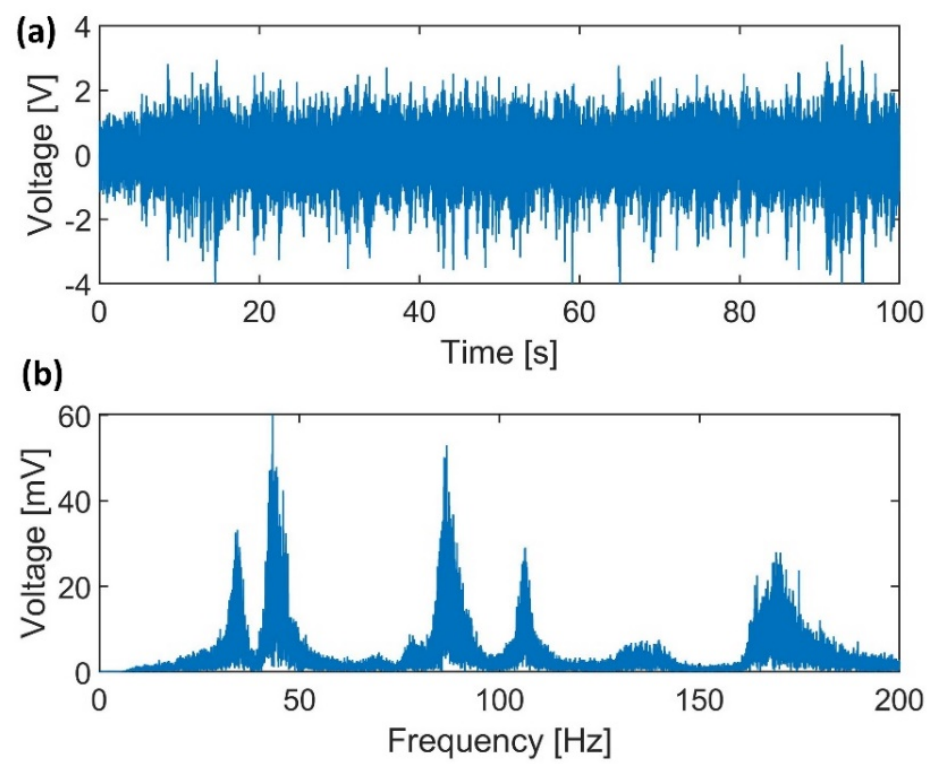

Fig 7: Pseudo-random test at $0.0058 \mathrm{~g}^{2} / \mathrm{Hz}$ from 5 to $400 \mathrm{~Hz}$, showing the broadband performance. (a) Timedomain output voltage; (b) frequency domain.

\section{Summary}

In this paper, a broadband energy harvester is designed and implemented using on-site operation vibration data collected from an underground train bogie. Vibrations over a broad bandwidth were observed from the on-site data, which requires the harvesters to be able to cover this range. A harvester using mechanical nonlinearity and multi-mode vibration is proposed within a unique design that consists of a pre-stretched hosting beam and multiple micro-beams deflected due to the repulsive magnetic forces at the free ends. The whole designed were implemented using piezoelectric beams with the dimensions of $26.5 \mathrm{~mm} * 1.5 \mathrm{~mm} * 0.5 \mathrm{~mm}$ and tested under pseudo-random conditions. The harvester illustrates a broadband feature between $10-130$ $\mathrm{Hz}$ with multiple peaks, showing its capability for the railway energy harvesting application.

\section{References}

[1] Weston, P., et al., Perspectives on railway track geometry condition monitoring from in-service railway vehicles. Vehicle System Dynamics, 2015. 53(7): p. 1063-1091.

[2] Hodge, V.J., et al., Wireless sensor networks for condition monitoring in the railway industry: A survey. 2014. 16(3): p. 1088-1106.

[3] Mitcheson, P.D., et al., Energy Harvesting From Human and Machine Motion for Wireless Electronic Devices. Proceedings of the IEEE, 2008. 96(9): p. 1457-1486.

[4] Beeby, S.P. and D. Zhu, Vibration energy harvesting: fabrication, miniaturisation and applications. SPIE Microtechnologies. Vol. 9517. 2015: SPIE.

[5] Gatti, G., et al., Harvesting energy from the vibration of a passing train using a single-degree-of-freedom oscillator. Mechanical Systems and Signal Processing, 2016. 66-67: p. 785-792.

[6] Wang, J., et al., Modeling on energy harvesting from a railway system using piezoelectric transducers. Smart Materials and Structures, 2015. 24(10): p. 105017.

[7] Fu, H. and E.M. Yeatman, Rotational energy harvesting using bi-stability and frequency up-conversion for low-power sensing applications: Theoretical modelling and experimental validation. Mechanical Systems and Signal Processing, 2019. 125: p. 229-244.

[8] Fu, H. and E.M. Yeatman, A methodology for low-speed broadband rotational energy harvesting using piezoelectric transduction and frequency up-conversion. Energy, 2017. 125: p. 152-161.

[9] Dhote, S., et al., Enhanced broadband multi-mode compliant orthoplanar spring piezoelectric vibration energy harvester using magnetic force. 2018. 135: p. 63-71.

Corresponding author: Hailing Fu, Wolfson School of Mechanical, Electrical and Manufacturing Engineering, Loughborough University, +44 (0)1509 222514, h.fu@lboro.ac.uk. 